always made progress possible again. Even after it has bcen recognised that we must accept an overall economy which is less lavish than before, great rebuilding will be needed. As the supplies of oil and gas run out the whole petroleum industry, for example, will have to be replaced by other industries, ultimately by ones that depend upon renewable resources. We have seen many such changes, for example the change over the past 50 years from coal to oil. These changes involve work and employment.

No growth overall need not mean overall stagnation once the correct direction is recognised and followed. Undisturbed Nature is stable, but it is certainly active. I therefore prefer the term renewal economy to no-growth society. The best chance to avoid ultimate breakdown and anarchy and to maintain any of the present forms of society is to take steps at once to change them. It should be noted that the change in philosophy must be farther reaching than a mere change between one or another existing political party or between a planned society and free enterprise. All the present economic philosophics ignore Nature's and mathematics' edict that growth cannot continue indefinitely. If some extreme governments have achieved conditions of no growth in their countries it is because of their ineptitude, not their desire.

The need is imperative to recognise and to start to implement this great revolutionary return from growth to stability. Its effects are likely to be more traumatic than those of any previous scientific revolution, but can best be ameliorated by early recognition and acceptance. Nearly three years have already passed since the revolutionary turning point in the availability of energy occurred. It is ironic that it would appear that it is the conservative blindness of those who should be leaders, whether in politics, business, or labour, that is the greatest impediment to advance.

\title{
Space: a new phase?
}

\section{Keith Runcorn and Paul Coleman describe plans for a major new effort in exploration and urge more European cooperation}

THE landings of Viking spacecraft on Mars on July 20 and September 4. 1976, and of Venera 9 and 10 on the surface of Venus in 1975, mark the culmination of a period of lunar and planetary exploration. Over the past decade manned Apollo landings and the unmanned Ranger, Surveyor, and Luna 16, 20 and 24 landings have been made on selected sites on the Moon. From the data collected on all these remarkable missions, our understanding of the evolution and present state of the Moon and planets is developing at an unprecedented rate. The next decade will see a revolution in our knowledge of the solar system comparable with those studies of continental drift and plate tectonics which, over the past two decades, have transformed our understanding of the Earth.

Europe, with a combined gross national product greater than that of the USA and the Soviet Union, has not so far taken a role which reflects its strength in this field, one of mankind's greatest explorations and scientific endeavours. An opportunity will be afforded by the International Solar System Programme, a project of international collaboration in the exploration of the solar system. The International Council of Scientific Unions recommended last year that all countries should consider how they can most effectively contribute.

Our knowledge of the Moon exceeds

Keith Runcorn is at the University of Newcastle-upon-Tyne. Paul Coleman is at the University of California, Los Angeles. in its detail that of any other body in the solar system except the Earth. While it might be feared that sampling the Moon in a restricted number of sites might hopelessly bias our interpretations, it is now clcar that the absence of an atmosphere and an ocean and the weaker tectonic forces than on Earth have resulted in much less segregation and concentration of elements and a much smaller variety of rock strata than on the Earth. This has in turn made it possible for the relatively small number of sampling sites to yield data reasonably representative of the Moon as a whole. On terrestrial planets limited samples might not be as successful in determining global properties.

The Moon differentiated about $4.4 \times 10^{9}$ years ago, forming an anorthositic gabbroic crust apparently thicker on the far side than the near side. The sources of the mare basalts. (crystallisation ages 3.2 to $3.8 \times 10^{4}$ years) which filled the great impact basins left in the Moon after catastrophic hombardment, seem to have differentiated at the same time. The processes that brought about this differentiation are obscure and the depth to which it extended is unknown. If the Moon is found to possess an iron core, this early differentiation must have occurred throughout the Moon. The siderophile and volatile elements on the Moon are depleted relative to the Earth and meteorites: information potentially a kcy to our understanding of the early evolution and origin of the Moon.

Three entirely unexpected charac-

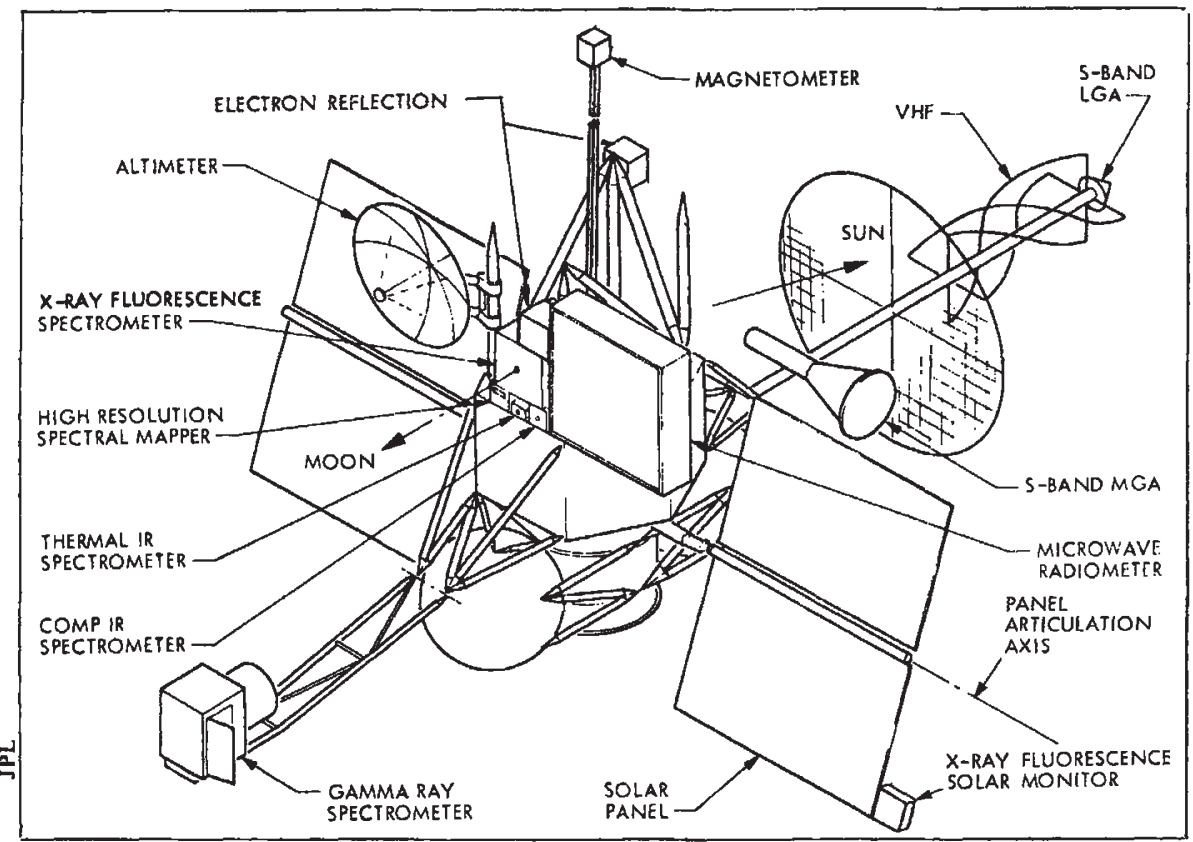

Flight configuration for $T B O L$ 
teristics were established from physical studies: large positive gravity anomalies over the circular mare (the mascons), moonquakes both deep and shallow, and widespread magnetisation of the crust. The interpretations of these discoveries are still controversial but we conclude that in its early history the Moon was much more active internally than it is today. If so, why has the Moon died?

The interpretation of gravity field on the near side requires an equally complete survey on the far side. Basins of comparable size to the mare are known to exist on the far side, but they are not filled with lava and whether they have negative or positive gravity anomalies is uncertain. Thus, the exact delineation of gravity above these farside basins is of critical importance to the understanding of the process which resulted in the flooding of mare and the production of mascons on the near side.

Again, the controversy concerning the interpretation of the magnetism of lunar rock centres upon whether the moon generated, between $4 \times 10^{\circ}$ years and $3.2 \times 10^{9}$ years ago, a general magnetic field which it does not have today. The direction of magnetisation of the lunar crust was impossible to determine by the methods used in terrestrial paleomagnetism; the Apollo samples were unoriented. Instead, interpretation of magnetic anomalies must be used. The existence of an iron core in which a dynamo process might once have been active remains in doubt. A more accurate value of the moment of inertia factor $(0.4$ for a uniform sphere) which is now given as $.392 \pm .003$ could resolve this particular matter.

The definitive solutions of many interesting problems raised by existing data depend on completing the survey of the moon which has been so well begun. To this end the lunar polar orbiter has been conceived in a mission named at present the Terrestrial Bodies Orbiter-Lunar (TBOL). A satellite carrying a variety of remote sensors would be put in lunar orbit about 100 kilometres above the surface so that a complete and relatively detailed sur-

\section{The experimental programme}

The geochemical experiments consist of a solid state (germanium) counter for gamma rays and four proportional counters (three looking at the moon and one at the sun) for $X$ rays. The former will detect both the radioactive nuclides $\mathrm{K}, \mathrm{Th}, \mathrm{U}$ and the elements $\mathrm{Fe}, \mathrm{O}, \mathrm{Si}, \mathrm{Ti}, \mathrm{Mg}, \mathrm{Al}$, $\mathrm{Ca}$ (in declining order of detectability) excited by the galactic cosmicray neutrons and by solar flares. The latter will detect the characteristic $X$ rays emitted from $\mathrm{Al}, \mathrm{Si}, \mathrm{Mg}$ for the quiescent sun and also $\mathrm{Ca}, \mathrm{K}, \mathrm{Fe}, \mathrm{Ti}$ during flares. The sampling depth will be $15 \mathrm{~cm}$ for the gamma rays and $10 \mu \mathrm{m}$ for the $\mathrm{X}$ rays and spatial resolutions will be $50 \mathrm{~km}$ and $10 \mathrm{~km}$, respectively. These experiments will provide much more detailed elemental abundance data than has so far been available and will contribute to the understanding of the differentiation of the moon, especially of the highland regions thought to be anorthositic gabbroic in character but only on the basis of fragmentary samples.

Water of the moon, perhaps retained in the regolith of deep craters near the poles, is exciting much interest and the gamma ray experiment may detect it from the 2.22 $\mathrm{MeV}$ line de-excitation of deuterium produced by slow neutrons created in material on the lunar surface by cosmic-ray bombardment.

The radioactivity is known to be greater in the western mare, so that an experiment to detect the surface heat flow is proposed. It may be surprising that this can be done from orbit, but microwave radiometers $(3 \mathrm{~cm}, 10 \mathrm{~cm}, 20 \mathrm{~cm})$-three identical beams with $50 \mathrm{~km}$ resolution-detect the black body radiation from different depths (down to about $5 \mathrm{~m}$ ) in the regolith where, because of its ex- tremely low conductivity, the vertical temperature gradient is large. These radiometers and infrared detectors will also measure the lunar daily in flow and outflow of solar radiation, from which the thermal conductivity of the regolith can be calculated. Thus the distribution of heat sources within the moon will be globally mapped and hypotheses such as convection in the interior will be tested.

Reffectance spectroscopy techniques over wavelengths $0.35-2.5 \mu \mathrm{m}$ will be used to determine the mineralogical composition. This method has already been used effectively to determine the composition of the major satellites of planets. The wavelength of absorption lines from the excitation of electrons from $\mathrm{Fe}^{++}, \mathrm{Fe}^{+++}$and ions of other transition metals are influenced by the crystalline structure of the SiO lattice. It will be supplemented by spectro-stereo images with six filters. The latter instrument, of course, will also provide, in high resolution $(\sim 100 \mathrm{~m})$, further details of the morphology, stratigraphy and, through crater counts, chronology of the lunar surface.

Magnetic maps of the moon will be obtained at the orbital altitude by a 3-component magnetometer accurate to $0.1 \gamma$ and at the surface by an electrostatic analyser of electron fluxes. The latter will detect low energy electrons, spiralling around the lines of the solar wind magnetic field, which are reflected from the convergence of the field lines produced by surface magnetic anomalies. These two methods will complement each other as crustal magnetic anomalies having sharp contrasts of magnetisation look very different at the surface and at heights of $100 \mathrm{~km}$. As various interesting explanations of the magnetisation of lunar rocks have been suggested, some involving cometary and meteoritic impacts, data on the general morphology of magnetic anomalies may establish whether or not these external sources or a magnetic field originating at the lunar core are responsible.

The existence of a lunar core can also be investigated by observing the electromagnetic response of the moon as it enters the fairly steady geomagnetic tail field. The core acts initially as diamagnetic and then the induced dipole moment begins to decay-from this initial decay the free decay time constant of the electric currents in the core can be determined.

The map of the gravity field on the near side will be improved by tracking of the orbiting satellite. For the far side of the moon, complete maps will be made of the gravity field, of which at present nothing is known except by inference from the evolution of the orbital elements of the subsatellites launched in the Apollo 15 and 16 missions. The closely related study of the figure of the moon has been controversial, as the coverage of the laser altimeters on the Apollo 15, 16 and 17 missions has been very limited and the classical earth-bound method, using the geometrical librations which enable height determinations to be made by the parallax principle, were extremely noisy. The radar altimeter in the orbiter should enable the surface to be mapped completely to a high accuracy. The moon departs from a sphere in a variety of ways and the mapping of the moon will be very important to refine our understanding of its non-hydrostatic shape. 
vey would be possible. A radio-relay satellite would be put into a more distant orbit to provide an almost continuous radio contact between the instrumented polar orbiter and the Earth. Such a mission, in which the scientific aspects have already been developed (see figure and box), is under consideration by NASA for a 1982 launch (see following story).

The Terrestrial Bodies Orbiter-Lunar mission is part of a new phase of planetary exploration, by means of remote-sensing orbiting spacecraft. A somewhat similar approach, but with a spinning spacecraft, is now under way in the United States for exploration of Venus, and a Jupiter orbiter is in the planning stages. These latter two missions will also involve atmospheric probes, which are unnecessary for planets and bodies without a substantial atmosphere. Modified versions of the TBOL spacecraft could extend further the exploration of such bodies in future.

More active European participation at this crucial stage in the exploration of the solar system would be timely and would enable European scientists, who contributed so much to the analysis of lunar samples, to revive the old scientific tradition of which we have been reminded in recent years by the anniversaries of Copernicus, Kepler and Galileo.

\section{Ford's parting gifts}

Colin Norman in Washington reports on the proposals for research and development in Mr Ford's third and final budget

WITH just three days of his brief and troubled Presidency left to run, $\mathrm{Mr}$ Ford this week presented Congress with a complex and detailed set of budget proposals for the 1978 Fiscal Year, which begins on October 1 , 1977. more than eight months after he leaves office. Though they are likely to be substantially revised, both by the incoming Carter Administration and by the Democratic-controlled Congress, $\mathrm{Mr}$ Ford's parting economic proposals could result in substantial increases in support for science and technology, particularly for basic research.

The proposals represent the lameduck Administration's detailed prescriptions for limiting growth in Federal spending, reforming the sprawling government bureaucracy and setting priorities among Federal programmes. During the next few weeks, President Carter and his economic advisers will sift through $\mathrm{Mr}$ Ford's budget and offer a raft of amendments to bring it into line with the new administration's economic and political philosophies. Carter will concentrate on the more political proposals, however, and it is likely that many of the less controversial programmes, which include most concerned with science and technology, will survive the change of administration more or less intact.

Mr Ford's budget includes a total of about $\$ 26,300$ million for research and development, an increase of about $8 \%$ over estimated expenditures this year. As in the past few years, defence and energy research and development account for the bulk of the increases, but basic research is also singled out for favourable treatment, rising by about $9 \%$, to reach some $\$ 3,000$ million. Inflation is expected to hover around $6 \%$, so the Ford budget proposals should result in a modest, but real, growth in Federal research support.

According to a discussion paper published by the Office of Management and Budget, funding for basic research in the United States has declined by about $27 \%$ over the past decade, chieffy because funding increases have not kept pace with inflation. Although the document suggests that basic science in the United States is competitive with that of other countries, the decline in support "has raised concerns about whether the United States might not be underinvesting in basic research to the detriment of the nation's future".

The chief beneficiary of the proposed increases is the National Science Foundation (NSF), scheduled for an increase in funds for some $12 \%$. According to Richard Atkinson, the acting director of NSF, a major use of the proposed increase will be to upgrade scientific instruments and equipment in universities and colleges. NSF's total budget is set to climb to $\$ 885$ million under Mr Ford's proposals.

The budget is the first to be produced since the establishment of the White House Office of Science and Technology Policy (OSTP), and some credit for the proposed increases is being claimed for the new arrangement. H. Guyford Stever, the head of OSTP and Mr Ford's science adviser (who will also leave the Federal Government this week) notes with some satisfaction that "although it is a constrained budget, it is not as constrained as in previous years". Asked whether he expects the Carter Administration to go along with the proposals, Stever pointed out that few of the research and development proposals have political implications, and he suggested that "if the incoming administration asks for advice on the research and development budget it would get much the same advice" as $\mathrm{Mr}$ Ford reccived. Since Mr Carter had not chosen a replacement for Dr Stever at the beginning of this week, he does not seem to feel an urgent need for such advice.
Included in the budget proposals are a number of new programmes which have been waiting in the wings for some time, and which are likely to meet with approval from the Carter Administration. Prominent among them is a major effort in earthquake research. A three-year project divided between the National Science Foundation and the Department of the Interior, it is expected to receive about $\$ 150$ million, with the first instalment of $\$ 23$ million coming this year. The goals are "development of a reliable prediction capability within 10 years through increased research on fundamental causes of earthquakes and precursor phenomena", establishment of building codes for structures in earthquake prone areas, publication of regional hazard assessment maps, and studies of the social. legal and economic implications of reliable earthquake prediction capability. Such a programme was recommended last year by a White House Advisory Committee, and a similar effort was incorporated in a bill passed last year by the Senate. The Carter Administration is unlikely to reject the proposal.

Another area to receive some longoverdue support in $\mathrm{Mr}$ Ford's budget is agricultural research. Some \$28 million has been proposed for a new programme of basic research on photosynthesis, nitrogen fixation, crop productivity, and genetic engineering of crop plants, funded through a system of competitive grants similar to those operated by the National Science Foundation and the National Institutes of Health. At present, virtually all the research supported by the Department of Agriculture is funded through block grants to institutions, and initiation of a competing grants programme has long been recommended, particularly by committees of the National Academy of Sciences, as a way to increase the quality of agricultural research. The proposed programme is virtually certain to receive a warm reception in Congress since the House last year approved a bill designed to initiate just such a programme. 